\title{
The role of jumps and options in the risk premia of interest rates
}

\author{
BRUNO LUND*
}

\begin{abstract}
There is evidence that jumps double the explanatory power of Campbell and Shiller (1991) excess bond returns' regressions (Wright \& Zhou, 2009), and options bring information about bond risk premia beyond that spanned by the yield curve (Joslin, 2007). In this paper I incorporate these features in a Gaussian Affine Term Structure Model (ATSM) in order to assess two questions: i) What are the implications of incorporating jumps in an ATSM for option pricing? and ii) How jumps and options affect the bond risk-premia dynamics? The main findings are: i) jump risk-premia is negative in a scenario of decreasing interest rates, and has a significant average magnitude of $1 \%$ to $2 \%$, which means that, it explains $10 \%$ to $20 \%$ of the level of the yields; ii) the Gaussian model (A30) and the Gaussian model with constant intensity jumps (A30J) are the ones that best fit the option prices; and iii) the Gaussian model with constant intensity jumps estimated jointly with options $(\mathrm{A} 30 \mathrm{~J})$ is the one that best identifies the risk premium.
\end{abstract}

Keywords: Jumps, option pricing, term structure, Poisson process, affine process, market price of risk, Asian options

JEL Codes: C11, C32, C51, G12, G13

\section{Introduction}

Understanding the behavior of the term structure of interest rates is important for both practitioners and policy makers. The former want to undertake profitable positions in bonds and interest rate derivatives and better assess interest rate risks, whereas the latter want to extract its economic content to guide policy decisions.

There is empirical evidence that both jumps and interest rate options are important to describe the risk premia behavior of interest rate. For instance, Wright and Zhou (2009) show that adding a measure of market jump volatility risk as an explanatory variable doubles the $R^{2}$ of the excess bond returns regressions

Submitted on 03 April 2014; Reviewed on 03 October 2018

* Head of Research and Modeling at Fundo Garantidor de Créditos.

- lundbru@gmail.com 
a la Campbell and Shiller (1991). Their result suggests that the addition of jump processes in a term-structure model might improve the understanding of the yield curve future behavior and its risk premia. In fact, Ludvigson and $\mathrm{Ng}$ (2009) find that real and inflation factors have important forecasting power for future excess returns in bonds beyond the one contained in the term structure. Jiang and Yan (2007) and Johannes (2004) show that jumps in interest rates are related to geopolitical events and surprises in macroeconomic releases. Combining these results suggests that jumps are somehow connected to the macroeconomy. In addition they indicate that Term Structure models that incorporate jumps in their underlying market price of risk are more suitable to analyze interest rates risk-premia dynamics and to evaluate option pricing.

On the other hand, Joslin (2007), Almeida, Graveline, and Joslin (2006), and Graveline (2006) show that options can bring information about risk premia beyond that spanned by interest rates only. In fact, a large number of papers include interest rate options in the estimation to better price risks in Term Structure Models (Almeida et al., 2006; Almeida \& Vicente, 2009a, 2009b; Graveline, 2006; Joslin, 2007). The use of options helps identify the market price of risk dynamics. Indeed, to the extent that options have information beyond interest rates, their inclusion will help estimate and capture state variables dynamics that drive future bond excess returns that are not spanned by the yield curve.

The aim of this paper is to study the inclusion of these two features (priced jumps and options data) in an Affine Term Structure Model (ATSM) framework to evaluate: i) what are the implications of incorporating jumps in an ATSM for option pricing, and ii) how jumps and options affect the bond risk-premia dynamics.

To answer the first question, I estimate three ATSMs without using options data: Gaussian with interest rates data only (A30), Gaussian with interest rates data only, random jumps and constant intensity (A30J), and Gaussian with interest rates data only, random jumps and time-varying intensity (A30JT). To assess question ii, I also estimate their counterparts using both options and interest rates data: A30o, A30oJ, and A30oJT.

The action plan is to compare the goodness of fitness of A30, A30J, and A30JT and then track the fit of the model-implied expectations of the interest rates against the 1-year ahead expectation released by the Central Bank of Brazil. 
To the extent that interest rates jump with Monetary Policy Committees (MPCs) and macroeconomic announcements, I expect that models with jumps will better price options. And, since there is evidence that options and jumps help identify and explain risk-premia, it is reasonable to conjecture that a model with jumps estimated jointly with options, will provide a better tracking of the market expectations and, consequently, a better identification of the risk premium dynamics. I also expect a significant contribution of jumps to risk-premia, since MPC decisions in Brazil provoke large discontinuous changes in the overnight rate. In summary, the objectives are twofold: to investigate model implied option prices, and the risk premium behavior generated by the inclusion of priced jumps estimated jointly with options. ${ }^{1}$

Other contributions are in the methodological framework on: i) how to price interest rate Asian options using a quasi-analytical formula when there is a compound Poisson process in one or more state variables of an Affine diffusion process; ii) how to estimate and filter for latent jumps on an Affine Jump Diffusion Term Structure model; and iii) how to decompose and isolate the jump component of the interest rates' risk premium.

The main findings are: i) jump risk-premia is negative in a scenario of decreasing interest rates, and has a significant average magnitude of $1 \%$ to $2 \%$, which means that, it explains $10 \%$ to $20 \%$ of the level of the yields; ii) the Gaussian model (A30) and the Gaussian model with constant intensity jumps (A30J) are the ones that best fit option prices; and iii) the Gaussian model with constant intensity jumps estimated jointly with options (A30oJ) is the one that best tracks the market 1-year ahead interest rates' expectations released by the Central Bank. This result suggests and reinforces the hypothesis that jumps and options help to better identify the risk premium dynamics.

Some related papers are Pan (2002), Jiang and Yan (2007), and Joslin (2007). The first uses an Affine Jump Diffusion (AJD) Model to examine the joint time series of the $S \& P 500$ index and its near-the-money short dated option. Her model is able to capture risk premia coming from both stochastic volatility and jumps. The distinguishing feature of my work is that I model jumps for fixed income instruments instead of stock indices. The second develops AJD and Quadratic Jump Diffusion models for the Term-Structure to investigate volatility risk premia. Even though it incorporates jumps it does not employ options data

${ }^{1}$ The risk premium is defined as the difference between the future interest rate expectations under the risk neutral measure and the physical measure. 
in the estimation and also does not study the impact of the inclusion of jumps in the risk premium of interest rates. Joslin (2007) studies weakly incomplete affine models for the term-structure to show that the inclusion of options in the estimation can bring information about volatility risk premia, and that a large fraction of it can be hedged using only bonds. However, it does not incorporate jumps and, as a consequence, has nothing to say about jump risk premia.

\section{Data Description}

To estimate the models, I use daily data from the nominal Brazilian Term Structure and the Asian IDI Interest Rate Options ranging from 2006, Jan $2^{\text {nd }}$ to 2007, Nov 23 ${ }^{\text {rd }}$. More specifically, I use the DI rate from the overnight Interbank loan market (similar to the LIBOR rate), registered at B3, as the 1 day spot interest rate to pin down the short-term of the yield curve, while the other maturities come from the DI future contracts traded at B3. By definition, the $n$-days maturity DI future contract quotes the fixed $n$-days spot rate priced by the market that breakevens the daily accrual of the overnight DI rate up to the maturity. It is of note that the DI future contracts don't trade at constant maturities, so that I interpolate the interest rate of all available maturities in each day of the sample to work with a constant-maturity interest rate dataset of $1,21,42,63,126,189,252,378$ and 504 working days $(w d){ }^{2}$ The graph at the top-left of Figure 1 shows evolution of the interpolated interest rates. And, the Figure 2 plots the Interbank DI interest rate. Note that it is practically a pure jump process.

The options data I use in the paper has the DI Index as the underlying asset. The DI Index is constructed by accruing the overnight Interbank DI interest rate daily. Note that it is not possible to know in advance what would be the future time $T$ DI Index with information up to time $t$, for $t \leq T$. Mathematically, the time $T$ DI Index is obtained by the following formula:

$$
I D I_{T}=\prod_{i=0}^{T-t-1} I D I_{t}\left(1+D I_{t+i, t+i+1}\right)^{1 / 252}
$$

The option contracts over the DI Index are negotiated between the counterparts and subsequently registered at the B3 over-the-counter market in terms of

${ }^{2}$ In Brazil, the majority of the traded financial instruments counts the passage of time by calculating the number of working days over $252\left(\frac{w d}{252}\right)$. 


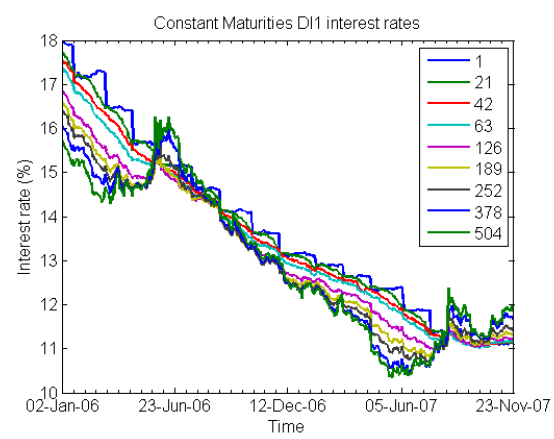

(a)

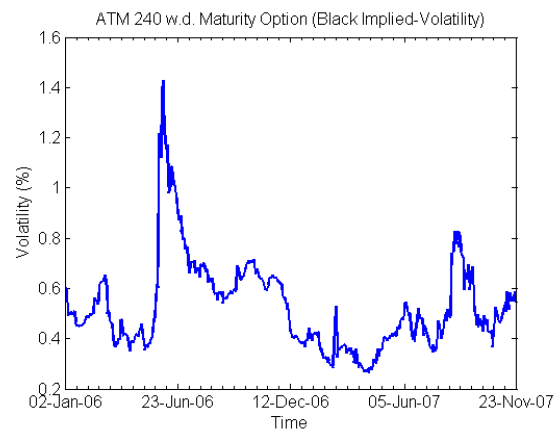

(c)

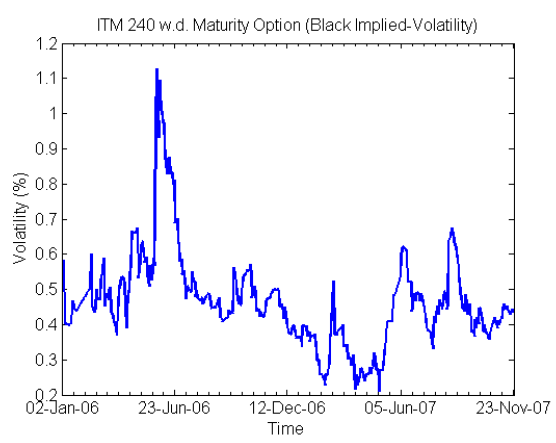

(b)

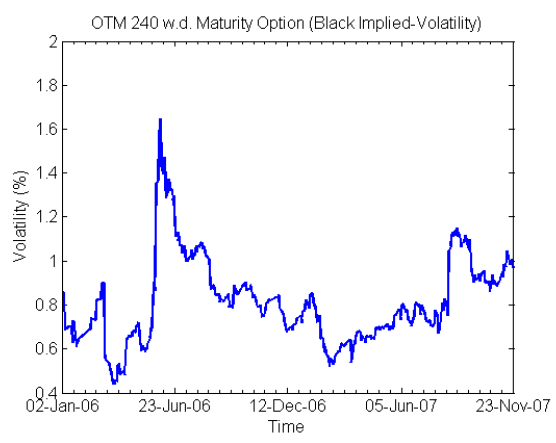

(d)

Figure 1. The daily evolution of the term structure of interest rate and of the volatility smirk for options with maturitiy 240 working days and moneyness of $(0.9,1,1.01)$.

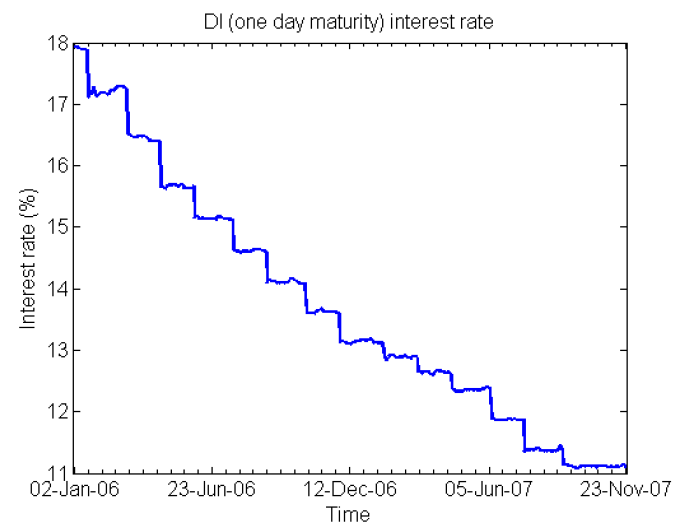

Figure 2. One day maturity Brazilian DI interest rate (annualized). 
the Black-implied volatility, strike price, maturity, option type (call or put), and execution rule (american or european). From the triple volatility-strike-maturity, I construct a constant daily moneyness-maturity volatility dataset to use in the paper by interpolating the volatilities of the call options in each day of the sample. The synthetic options constructed by the interpolation procedure have moneyness equal to $0.99,1$ and 1.01, and maturity of 240 working days. I define moneyness as:

$$
\text { Moneyness } \triangleq \frac{V P(\text { Strike })}{\text { Price }},
$$

where $V P(x)$ is the present value of $x$.

Some other studies, as Duffie, Pan, and Singleton (2000), define moneyness as the strike-price ratio, but I'd rather use the definition in (2) because it classifies the moneyness of the options in a more precise manner. To see that, note that, for call (put) options, the moneyness is equal to one if the option is at-the-money, less than one if in-the-money (out-the-money), and higher than one if out-the-money (in-the-money). The graphs at the top-right and the two at the bottom of Figure 1 show, respectively, the interpolated volatilities for the in-, at- and out-the-money options wiwth maturity of 240 working days. As expected, the volatility decreases with the call option moneyness, that is, from the out-the-money (OTM) to the in-the-money (ITM) options.

I estimate several models using either joint option-term structure data or only yield curve data. For the richer dataset, I only include at- and out-the-money options. The reason not to use in-the-money data in the estimation is that there are too few deals at this level of moneyness. To the extent this could mean a potentially poor price formation, a feature didn't want influencing the model results, I decided not to use the ITM options to estimate the models in which the options data are used.

The B3 quotes the IDI options in terms of Black implied volatility. To calculate the IDI option prices and to keep them comparable, I use the Black Model under the assumption the underlying asset is at 100,000 in each day of the sample.

\section{Model}

The theoretical model and the bond pricing formula follow Duffie et al. (2000) and appear in the Appendix A. 


\subsection{The market price of risk}

Suppose that the parameters of $(38)$ are given by $\Theta^{\mathrm{Q}}=\left(K^{\mathrm{Q}}, H^{\mathbb{Q}}, \lambda^{\mathrm{Q}}, \theta^{\mathbb{Q}}\right)$ under the risk-neutral measure $\mathbb{Q}, \Theta^{\mathbb{P}}=\left(K^{\mathbb{P}}, H^{\mathbb{P}}, \lambda^{\mathbb{P}}, \theta^{\mathbb{P}}\right)$ under the physical measure $\mathbb{P}$, and $\Theta^{\mathbb{P}^{\mathrm{MB}}}=\left(K^{\mathbb{P}}, H^{\mathbb{P}}, \lambda^{\mathbb{Q}}, \theta^{\mathbb{Q}}\right)$ under the brownian motion physical measure $\mathbb{P}^{\mathrm{MB}}$. Then, the relation between these measures are given by the following Radon-Nikodym derivative:

$$
\frac{\mathrm{d} \mathbb{P}}{\mathrm{d} \mathbb{Q}}=\frac{\mathrm{d} \mathbb{P}}{d \mathbb{P}^{\mathrm{MB}}} \times \frac{\mathrm{d} \mathbb{P}^{\mathrm{MB}}}{\mathrm{d} \mathbb{Q}}
$$

The first term defines the change of measure of Poisson Processes that determines the jump risk-premia, whereas the second one the change of measure of Brownian Motions that drives the diffusion risk-premia. For more details see the Appendix B.

\subsection{Pricing Asian Options}

Here I show how to price Asian options that depend on the path of the short-term interest rate. Here, the path dependence will be denoted by the integral in time of the instantaneous interest rate:

$$
Y(t, T)=\int_{t}^{T} r(u) \mathrm{d} u .
$$

The DI Index can be approximated by the following equation:

$$
I D I(T)=I D I(t) e^{Y(t, T)} .
$$

It's possible to show that this approximation is a very good one.

The IDI call option has the following payoff structure:

$$
\begin{aligned}
C(K, t) & =\mathbb{E}\left\{e^{-Y(t, T)}[I D I(T)-K]^{+} \mid \mathcal{F}_{t}\right\}, \\
C(k, t) & =\mathbb{E}\left\{\left[I D I(t)-e^{k} e^{-Y(t, T)}\right]^{+} \mid \mathcal{F}_{t}\right\}
\end{aligned}
$$

where $k=\log (K)$ and for now on I will use $y=Y(t, T)$.

To price the IDI option, define the Fourier Transform $\mathcal{G}(\lambda, k)$ as

$$
\mathcal{G}(\lambda, k)=\mathbb{E}\left[e^{-y \lambda} I_{y \geq k-i d i}(k) \mid \mathcal{F}_{t}\right]
$$


Then, the option price is given by

$$
C(k, t)=I D I(t) \mathcal{G}(0, k)-e^{k} \mathcal{G}(1, k) .
$$

Note that $\mathcal{G}(0, k)=P^{\mathbb{Q}}\left(Y \geq k-i d i \mid \mathcal{F}_{t}\right)$ and $\mathcal{G}(\lambda, k)=c P^{\mathbb{F}}\left(Y \geq k-i d i \mid \mathcal{F}_{t}\right)$ where $i d i=\log (I D I(t))$, the Radon-Nykodim derivative is given by $\frac{\mathrm{dF}}{\mathrm{dQ}}=$ $e^{-\lambda y} / \mathbb{E}\left(e^{-\lambda y} \mid \mathcal{F}_{t}\right)$ and $c=\mathbb{E}\left(e^{-\lambda y} \mid \mathcal{F}_{t}\right)$. Using this change of measure the option price can be rewritten as

$$
C(k, t)=I D I(t) P^{\mathbb{Q}}\left(Y \geq k-i d i \mid \mathcal{F}_{t}\right)-e^{k} P(t, T) P^{\mathbb{F}}\left(Y \geq k-i d i \mid \mathcal{F}_{t}\right) .
$$

For a fixed $\lambda$, the generalized Fourier Transform of $\mathcal{G}$ is

$$
\hat{\mathcal{G}}(\lambda, u)=\int_{-\infty}^{+\infty} e^{i u k} \mathrm{~d} \mathcal{G}(\lambda, k) .
$$

Solving the Levy Integral, we have

$$
\hat{\mathcal{G}}(\lambda, u)=-\mathbb{E}\left[e^{-y(\lambda-i u)+i u \cdot i d i} \mid \mathcal{F}_{t}\right]
$$

It hen follows from the Radon-Nikodym derivative that

$$
\hat{\mathcal{G}}(\lambda, u)=-\mathbb{\mathbb { Q }}\left[e^{-y \lambda} \mid \mathcal{F}_{t}\right] \times \mathbb{E}\left[\mathbb{E}\left[e^{i u y+i u \cdot i d i} \mid \mathcal{F}_{t}\right]\right.
$$

Defining

$$
I=\int_{-\infty}^{+\infty} \frac{e^{-i u x} \hat{\mathcal{G}}(\lambda, u)}{i u} \mathrm{~d} u .
$$

it's possible to show that

$$
\mathcal{G}(\lambda, x)=\frac{\mathcal{G}(\lambda,-\infty)}{2}-I \frac{1}{2 \pi}
$$

where $\mathcal{G}(1,-\infty)=P(t, T)$ and $\mathcal{G}(0,-\infty)=1$.

According to Joslin (2007), under the measure $\mathbb{F}, y$ is roughly normally distributed. More precisely, $\hat{\mathcal{G}}$ can be rewritten as

$$
\hat{\mathcal{G}}(\lambda, u) \approx c \times e^{i u \mu_{y}-\frac{1}{2} u^{2} \sigma_{y}^{2}}
$$

The Gaussian behavior under $\mathbb{F}$ happens because, despite the ratio of the 
Levy integrand is not constant (i.e. they are not the same), there is no huge variation, particularly in the area that is relevant for computing the integral numerically.

The implication of this result is that I only need to compute the first and second moments to calculate the IDI option price.

As opposed to the Fast Fourier Transform method of Carr and Madan (1999) this procedure is quite fast and allows pricing options of different moneyness in the whole time-series. Collin-Dufresne and Goldstein (2002) also present an approximated method based on cumulants to price swaptions in a fast way, but according to Joslin (2007) is less accurate and slower than the procedure presented here. Other works that price IDI options using different methods are Almeida and Vicente (2006, 2009a, 2009b).

\subsubsection{Change of Measure}

Fix $\lambda=1$, then the Radon-Nikodym derivative is given by

$$
M(t)=\frac{\mathrm{d} \mathbb{F}}{\mathrm{d} \mathbb{Q}}=\frac{e^{-y}}{\mathbb{E}\left(e^{-y} \mid \mathcal{F}_{t}\right)}=\frac{e^{-y}}{P(t, T)}=e^{-y} e^{-A(\tau)-B(\tau) X(t)},
$$

where $\tau$ is the time to maturity of the option, $T=t+\tau$, and $A(\tau)$ and $B(\tau)$ are the solutions of the Ricatti's ODE.

By the Martingal Representation Theorem, one can find the market price of risk $\theta$ that relates $\mathbb{F}$ to $\mathbb{Q}$ by using the differential operator in $M(t)$ :

$$
\mathrm{d} M(t)=-M(t) \theta(t)^{T} \mathrm{~d} W^{\mathbb{Q}}(t)
$$

where,

$$
\theta(t)=\Sigma(X(t), t)^{T} B(\tau)
$$

Girsanov Theorem gives the relation between Brownian motions under different measures:

$$
\mathrm{d} W^{\mathbb{F}}(t)=\theta(t) \mathrm{d} t+\mathrm{d} W^{\mathbb{Q}}(t) .
$$

Combining the results of these two theorems one can show that the state vector under the measure $\mathbb{F}$ follows

$$
\mathrm{d} X(t)=\left(K_{0}^{\mathbb{F}}+K_{1}^{\mathbb{F}} X(t)\right) \mathrm{d} t+\Sigma(X(t), t) \mathrm{d} W^{\mathbb{F}}(t),
$$


with

$$
\begin{aligned}
K_{0}^{\mathrm{F}} & =K_{0}^{\mathrm{Q}}-\alpha \odot B(\tau), \\
K_{1}^{\mathrm{F}} & =K_{1}^{\mathrm{Q}}-\beta \odot B(\tau) \iota^{T}, \\
\Sigma(X(t), t) & =\sqrt{\operatorname{diag}(\alpha+\beta X(t))},
\end{aligned}
$$

where $\odot$ is defined as the element by element product.

\section{Empirical Analysis}

\subsection{Estimation Procedure}

In order to estimate the models I solve for $X(t)$ using the linear bond yields relation (43) (see Appendix A.1) for the maturities 1, 126 and 378 working days to find the factors

$$
X(t)=\bar{B}^{*-1}\left[y^{*}(t)-\bar{A}^{*}\right]
$$

where $\bar{B}^{*}=[\bar{B}(1), \bar{B}(126), \bar{B}(378)]^{T}, \bar{A}^{*}=[\bar{A}(1), \bar{A}(126), \bar{A}(378)]^{T}$, and $y^{*}(t)=$ $[y(1, t), y(126, t), y(378, t)]^{T}$.

The vector of yields $y^{*}(t)$ used in the inversion are assumed to be priced without error, while the other yields $y^{* *}(t)$ and options $C^{* *}(t)$ are assumed to be priced with Gaussian independent errors. Note that I use the notation * for yields that are exactly priced, and ${ }^{* *}$ for yields and options that are price with error, and no star for actual yields and option prices $(y(t), C(t))$.

I estimate six models to evaluate: i) what are the implications of incorporating jumps in an ATSM for option pricing, and ii) how jumps and options affect the bond risk-premia dynamics. To answer the first question, I estimate three ATSMs without using options data: Gaussian with interest rates data only (A30), Gaussian with interest rates data only, random jumps and constant intensity (A30J), and Gaussian with interest rates data only, random jumps and time-varying intensity (A30JT). To assess question ii, I also estimate their counterparts using both options and interest rates data: A30o, A30oJ, and A30oJT. For more details on the specifications, see Appendix C.

For the A30, A30J and A30JT models I use the constant-maturity interest rate dataset of $1,21,42,63,126,189,252,378$ and 504 working days, whereas for the remaining models I use also the ATM and OTM options data with maturity of 240 working days. 
To construct the likelihood of the models, I assume that only one jump can happen by the end of each day, that is, I approximate a Poisson process by a Binomial process. With this hypothesis the time- $t$ likelihood is given by a mixture of two normal distributions, with weights given by the jump intensity $\lambda(X(t))$ :

$$
\begin{aligned}
l_{t}(\Theta)=(1- & \lambda(X(t-1)) h) f(y(t) ; \Theta \mid N(t)-N(t-1)=0) \\
+ & \lambda(X(t-1)) h f(y(t) ; \Theta \mid N(t)-N(t-1)=1)
\end{aligned}
$$

where $h=\frac{1}{252}$ and $f_{t}=f(y(t) \mid N(t)-N(t-1))$; and

$$
\begin{aligned}
f_{t} & =f\left(y^{*}(t) \mid N(t)-N(t-1)\right) \times f\left(y^{* *}(t) \mid N(t)-N(t-1)\right) \\
& =\left|\bar{B}^{*}\right|^{-1} f(X(t) \mid X(t-1), N(t)-N(t-1)) \times f\left(y^{* *}(t)\right)
\end{aligned}
$$

is a multivariate normal probability density function (pdf).

I use a MCMC Metropolis Hasting algorithm to estimate all the models. I draw 100,000 set of parameters from the posterior distribution and use the mean estimate of the last 5,000 replications.

\subsection{Model implied option pricing and state variables' factors}

\subsubsection{Option pricing}

I first compare the models that I estimate without using derivatives to evaluate which entails the best fit to the IDI call options prices. Figure 3 plots the evolution of the ITM, ATM and OTM IDI call prices of maturity $240 \mathrm{wd}$ for all estimated models against the actual price. A30 is the best in terms of mean absolute pricing errors, but the A30J better captures extreme spike behaviors on prices and volatilities. This happens because including jumps creates another source of variation that captures such sudden and extreme moves.

Unexpectedly A30JT overpriced the OTM, ATM and ITM options. This probably happens because the time varying intensity absorbs any change in volatility, while in the other models this variation ends in the residuals. Since volatilities are positively related to prices, overpricing arises.

I also plot in Figure 3 the models that incorporate options in the estimation (A30o, A30oJ and A30oJT), one should note there is a trade-off between the goodness of fit of rates and ATM options versus rates and OTM options. The 


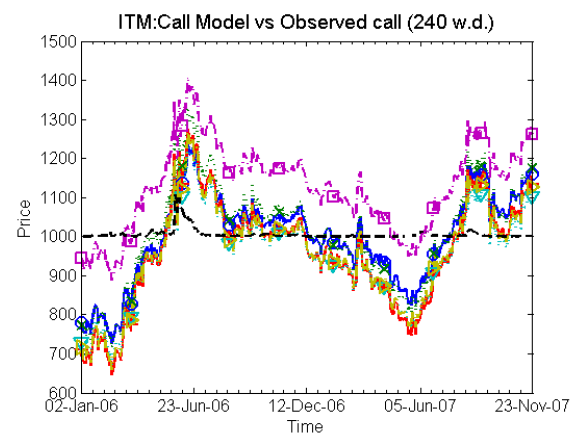

(a)

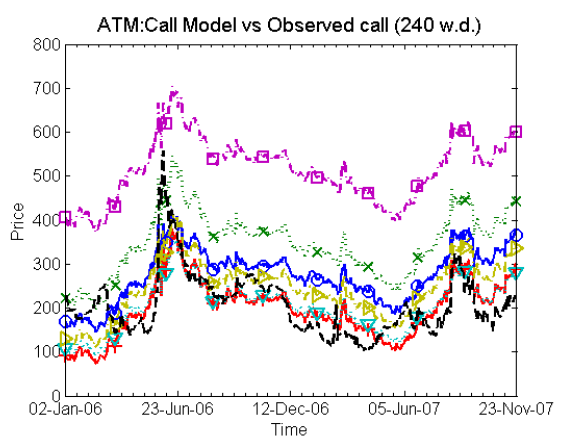

(b)

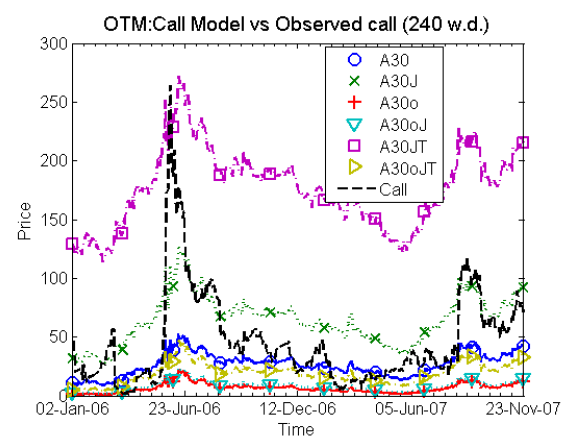

(c)

Figure 3. The daily evolution of the implied call IDI option prices with maturity 240 working days and moneyness of $(0.99,1,1.01)$.

ATM derivative has a better model price than its OTM counterpart. This happens because, to the extent ATM option prices are higher than the OTM prices, minimizing absolute errors in the estimation reflects on lower ATM relative pricing errors than OTM ones. Consequently, none of the three models is able to capture the huge spikes on the OTM option prices around June-2006 and September-2007.

\subsubsection{State variables' factors rotation}

Interpreting the latent state variables' factors presented in Figure 4 is not easy, because they are the result of an inversion of the system (25) presented in subsection 4.1. Note yet that their loadings are not fixed and depend on the estimation result.

However, it is possible to interpret the latent state variables by projecting 


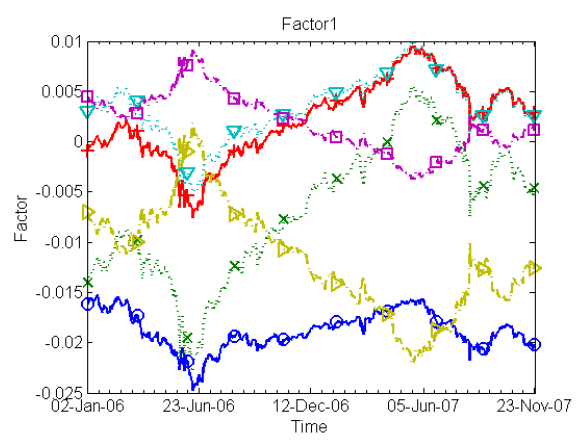

(a)

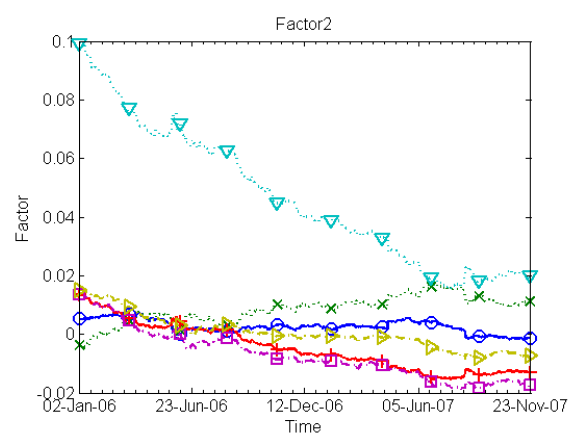

(b)

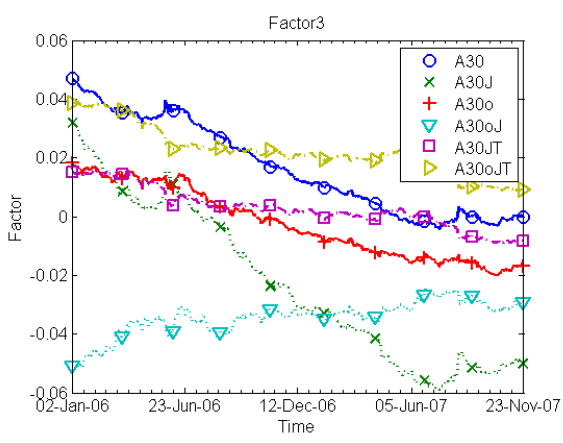

(c)

Figure 4. The daily evolution of the three factors $X(t)=\bar{B}^{*-1}\left[y^{*}(t)-\bar{A}^{*}\right]$ inverted from yields of maturities 1, 126 and 378 .

them on the space generated by the empirical factors of: level $(L(t))$, slope $(S(t))$ and curvature $(C(t))$. I construct the space of empirical factors as it follows:

$$
\begin{aligned}
L(t) & =\frac{y(63, t)+y(252, t)+y(504, t)}{3} \\
S(t) & =y(504, t)-y(63, t) \\
C(t) & =y(504, t)+y(63, t)-2 \cdot y(252, t),
\end{aligned}
$$

and project the latent variables in it by running regressions with the model implied factors as explained variables and the empirical factors as the explanatory variables:

$$
X_{i}(t)=a+b_{1} L(t)+b_{2} S(t)+b_{3} C(t)+\varepsilon_{i}(t) .
$$

The results of the regressions are plotted in Figure 5. Note that in all models 


\section{Bruno Lund}

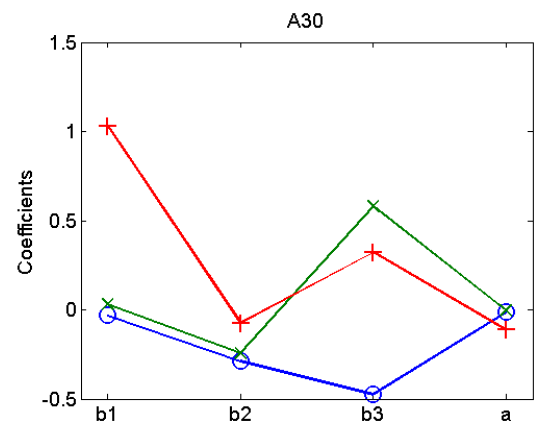

(a)

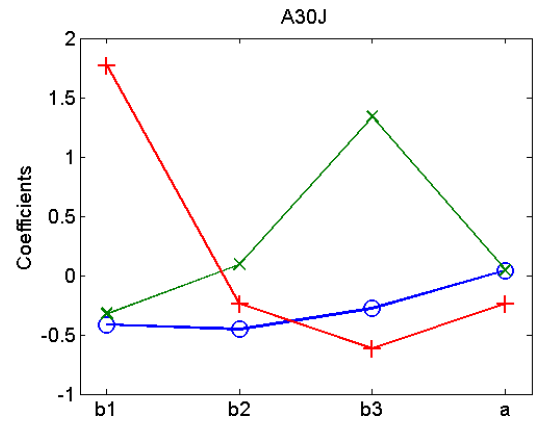

(c)

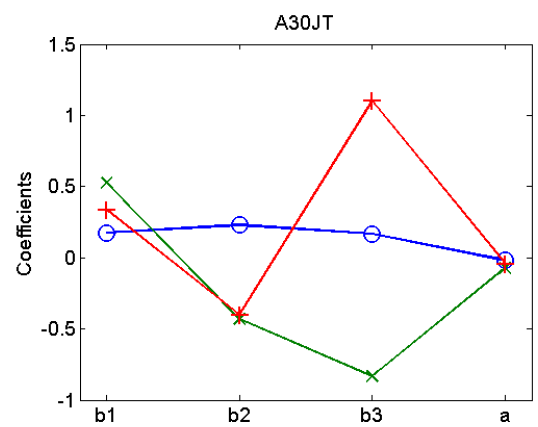

(e)

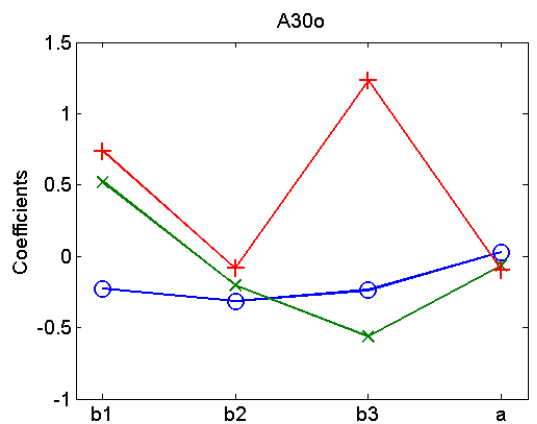

(b)

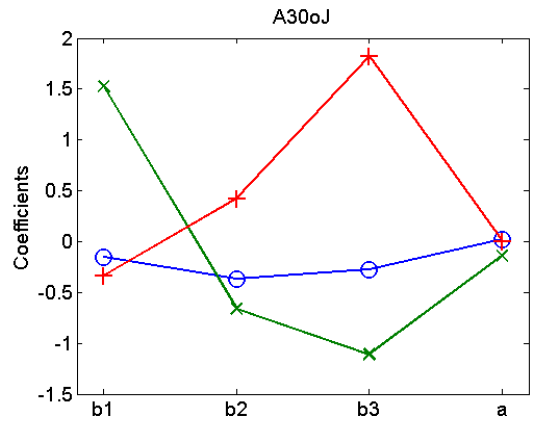

(d)

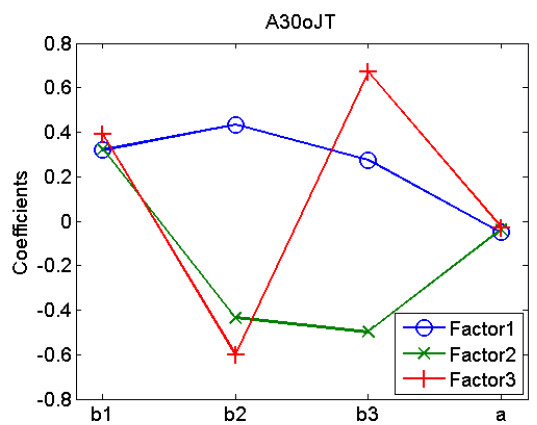

(f)

Figure 5. The coefficients of the factor regressions done as in equation (31) for the six models. 
(except for the A30) a shock in factor 1 affects all the empirical factors by the same amount. In the models without jumps (top two graphs), a shock in factor 3 affects positively the level and curvature, but the first is more important in the model estimated without options. This bigger curvature loading happens in all implied factor 3 from the models that use options in the estimation. One possible explanation for it is that curvature is closely related to variance, and the use of options allows to better identify volatility. Interestingly, contrary to the models that do not present jumps, the factors of the models with jumps are almost equal to the ones with options and jumps. Note that, in the constant intensity models, there is a switch between factor 3 and factor 2 from the model without options to the one including options, but once adjusting for that, they have almost the same loadings. This suggests that models with jumps identify better the factors.

\subsubsection{Jumps and Monetary decisions}

In my specification (see Appendix C), in order to identify the jumps I impose that factor 3 only is allowed to jump. One interesting consequence of that specification is that it is possible to evaluate the probability of interest rate jumps by decomposing this factor in two: i) a continuous part $X_{3}^{c}(t)$ that drives small movements in the interest rates; and ii) a jump part $N(t)$ responsible to capture discontinuous moves of interest rates and that can be related to a news announcement surprising the market affecting the interest rates in a discontinuous manner. One example is a monetary policy committee decision to change the target interest rate:

$$
X_{3}(t)=X_{3}^{c}(t)+N(t)
$$

Using that decomposition one can filter out the probability of one jump-event in the interest rates between periods $t$ and $t-1$ given all available information of the yields, $y(t)$, up to time $t, P(N(t)-N(t-1)=1 \mid Y(t))$. Note that higher probability of jumps are related with news announcements affecting significantly the interest rates. To see that I presented in Figure 6 the probability of jumps for the models with constant intensity (line) and the changes in the Selic interest rate target (circles) decided in monetary policy committees. Note that, even though, there are more jumps filtered in the model with options, changes in the Selic target are more closely tracked by the model without options. One possible explanation for that is that jumps are also linked to several geopolitical events and 


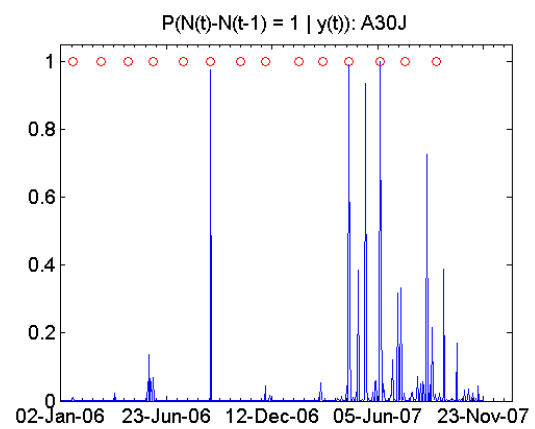

(a)

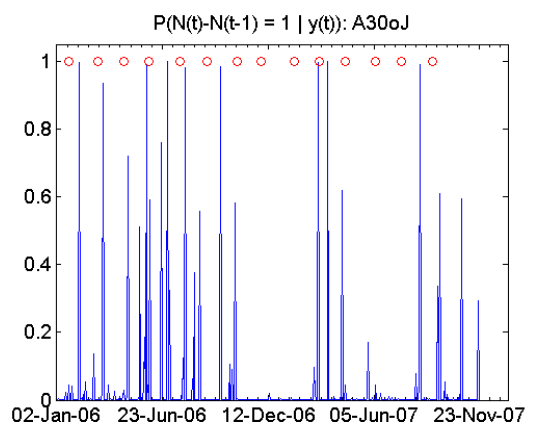

(b)

Figure 6. Circles are changes in the Selic monetary policy interest rate target. The line is the probability of one jump-event in the third state between periods $t$ and $t-1$ given all available yields information $y(t)$ up to time $t, P(N(t)-N(t-1)=1 \mid Y(t))$.

surprises in macroeconomic releases, unrelated with monetary policy decisions, that generally increase the uncertainty over the economy. As options captures uncertainty through market volatility better than bonds, jumps unrelated to policy decisions are better filtered in models estimated with option data.

The next section shows the methodology I use to theoretically decompose the interest rate risk premium.

\section{$5 \quad$ Risk Premium Decomposition and Identification}

Long term rates are equal to the average future expected short-term interest rates plus a risk premium amount. Therefore, if someone takes expectations under the risk neutral and the physical measure, it will attain two distinct values. The term premium is the gap between physical and risk neutral expectations.

Joslin (2007) explores this feature to decompose the $T$-year zero coupon yield $y(t, T)$ from a stochastic volatility model without jumps into tree components: i) physical expectation of future interest rates, ii) convexity, and iii) risk premia. I extend the Joslin risk premia decomposition to include a jump component by splitting the component iii into two: the jump risk premia and the Brownian risk premia. The first absorbs the risk premium coming from discontinuous variations in the interest rates, while the second the risk premium arising from small continuous variations in the interest rates. 
Differently from Joslin (2007), I use three measures $\left(\mathbb{Q}, \mathbb{P}^{\mathrm{MB}}, \mathbb{P}\right)$ (see subsection 3.1) to decompose the risk premium. To isolate the risk premium related to jumps one can take the difference between the expectations under the measures $\mathbb{Q}$ and $\mathbb{P}^{\mathrm{MB}}$ and to calculate the risk premium related to small and continuous variations in the prices one should take the difference between the expectations under the measures $\mathbb{P}$ and $\mathbb{P}^{\mathrm{MB}}$. The extended decomposition is presented in equations (34) to (37):

$$
\begin{aligned}
y(t, T) & =y^{E}(t, T)+y^{B R P}(t, T)+y^{J R P}(t, T)+y^{C}(t, T), \\
y^{E}(t, T) & =\frac{1}{T} \int_{t}^{t+T} \mathbb{E}^{P}[r(u)] \mathrm{d} u, \\
y^{B R P}(t, T) & =\frac{1}{T} \int_{t}^{t+T}\left(\mathbb{E}^{\mathrm{MB}}[r(u)]-\mathbb{E}^{P}[r(u)]\right) \mathrm{d} u, \\
y^{J R P}(t, T) & =\frac{1}{T} \int_{t}^{t+T}\left(\mathbb{E}^{Q}[r(u)]-\mathbb{E}^{\mathrm{MB}}[r(u)]\right) \mathrm{d} u, \\
y^{C}(t, T) & =\frac{1}{T}\left(\ln \mathbb{E}^{Q}\left[\exp \left\{\int_{t}^{t+T} r(u) \mathrm{d} u\right\}-\int_{t}^{t+T} \mathbb{E}^{Q}[r(u)] \mathrm{d} u\right) .\right.
\end{aligned}
$$

Figure 7 plots all the terms of the decomposition. Note first, that the Brownian premium of the models estimated with options in comparison with the ones estimated using only yields present higher levels of premium. This difference is more prevalent at the end of the sample (except for the models with time-varying intensity). Moreover, it is noticeable the opposite behavior for the 1-year yield expectation component. Second, models with options have more flat risk premiums, indicating a greater adherence to the weak form of the expectation hypothesis, in which the risk premium is constant. ${ }^{3}$

The jump risk premium components are always negative, ranging between -80 to -190 bps. At first sight, this result seems to be at odds with the intuition that jumps in interest rates are risky and, therefore, should have a positive premium. However, in the sample period, jump sizes have a negative mean $\left(\mu_{J}\right)$, so that the arrival of a jump is on average good news to a bond investor.

Another finding is that the negative jump premia is compensated by higher

\footnotetext{
${ }^{3}$ Long yields are equal to future expected short rates plus a constant risk-premium (that can vary with bond maturity).
} 


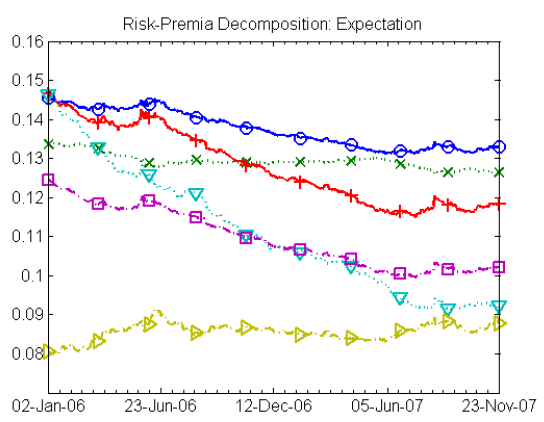

(a)

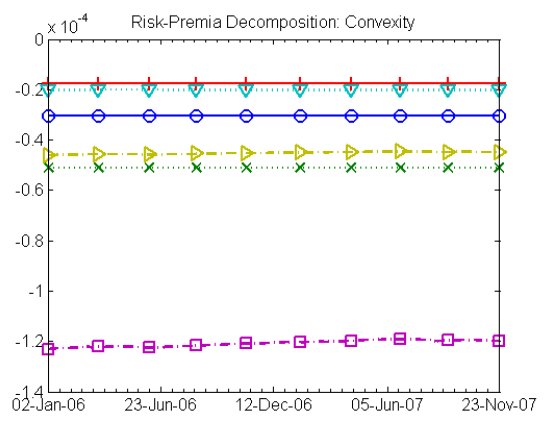

(c)

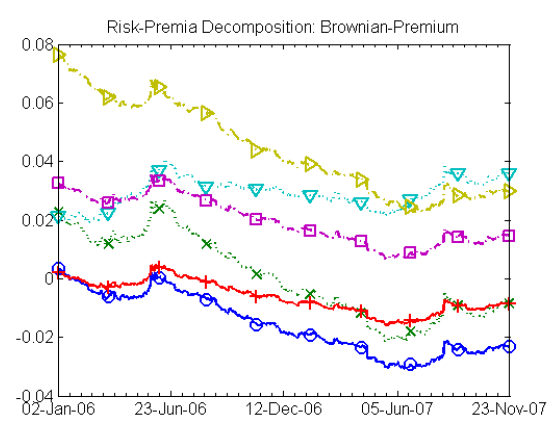

(b)

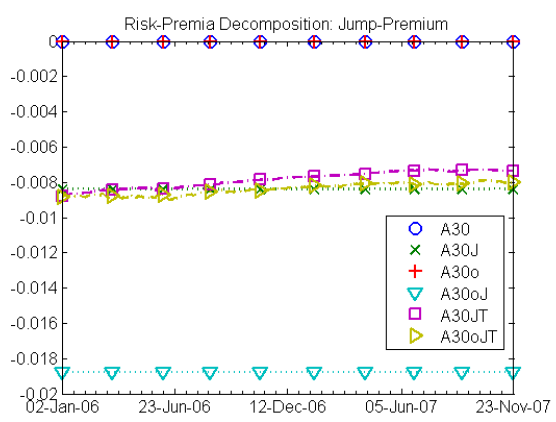

(d)

Figure 7. The daily evolution of the one year risk premiums, expectation and Convexity measures given by equations (34)-(37).

levels of brownian premium. This fact can be seen when comparing models with jumps with theirs counterparts that do not jump (see graphs in the right of Figure 7). Probably, within a larger sample that includes both negative and positive jumps, this result would change and turn the sign of the jump premia.

The convexity terms are very small and influence the actual yields in less than 1.5 basis point, but interestingly models estimated using interest rates only have a larger convexity in absolute values than their counterparts estimated jointly with options.

The most interesting result regarding risk premium arises from the comparison between the 1-year ahead interest rate model expectations' paths and the average 1-year ahead expectations released by the Central Bank of Brazil. ${ }^{4}$ The model

${ }^{4}$ See https://www3.bcb.gov.br/expectativas/publico/consulta/serieestatisticas for interest rates' expectations data released by the Central Bank of Brazil. 
with constant intensity jumps estimated jointly with options, A30oJ, is by far the model that best tracks the market players expectations released by the Central Bank in terms of mean absolute error. This finding is paramount, because to the extent that the models fitted the level of interest rates, then the model that best capture the market expectations is also the one that best describes the risk premium. This result is a strong one and corroborates the hypothesis that models with jumps and jointly estimated with options are better able to identify the risk premium dynamics.

\section{Conclusion}

In this paper I find that jump risk-premia is negative in a scenario of decreasing interest rates, explaining $10 \%$ to $20 \%$ of the level of the yields. I also show that Gaussian models without jumps or with a constant jump intensity price relatively well option prices, whereas employing the latter estimated jointly with options data is paramount if one wishes to track the risk premium over time. These results corroborate the hypothesis that jumps and options help identify the risk premium dynamics.

\section{References}

Almeida, C. I. R., Graveline, J., \& Joslin, S. (2006). Do interest rate options contain information about excess returns.

Almeida, C. I. R., \& Vicente, J. (2006). Pricing and hedging Brazilian fixed income options. In $6^{o}$ Encontro Brasileiro de Finanças, Vitória, ES. Retrieved from http://bibliotecadigital.fgv.br/ocs/index.php/ebf/6EBF/ paper/view/1379

Almeida, C. I. R., \& Vicente, J. (2009a). Are interest rate options important for the assessment of interest rate risk. Journal of Banking $\&$ Finance, 33(8), 1376-1387. http://dx.doi.org/10.1016/j.jbankfin.2009.02.003

Almeida, C. I. R., \& Vicente, J. (2009b). Identifying volatility risk premia from fixed income Asian options. Journal of Banking \& Finance, 33(4), 652-661. http://dx.doi.org/10.1016/j.jbankfin.2008.11.011

Campbell, J. Y., \& Shiller, R. J. (1991). Yield spreads and interest rate movements: A bird's eye view. The Review of Economic Studies, 58(3), 495-514. http://dx.doi.org/10.2307/2298008

Carr, P., \& Madan, D. B. (1999). Option valuation using the fast Fourier transform. J. Comp. Finance, 2(4), 61-73. http://dx.doi.org/10.21314/ JCF.1999.043 
Cheridito, P., Filipović, D., \& Kimmel, R. L. (2007). Market price of risk specifications for affine models: Theory and evidence. Journal of Financial Economics, 83(1), 123-170. http://dx.doi.org/10.1016/j.jfineco.2005 .09 .008

Collin-Dufresne, P., \& Goldstein, R. S. (2002). Pricing swaptions within an affine framework. The Journal of Derivatives, 10(1), 9-26. http://dx.doi.org/ 10.3905/jod.2002.319187

Duffie, D., Pan, J., \& Singleton, K. (2000). Transform analysis and asset pricing for affine jump-diffusions. Econometrica, 68(6), 1343-1376. http:// dx.doi.org/10.1111/1468-0262.00164

Graveline, J. J. (2006). Exchange rate volatility and the forward premium anomaly. Retrieved from http://www.nccr-finrisk.uzh.ch/media/pdf/ GravelineJMP.pdf

Jiang, G. J., \& Yan, S. (2007, July). Affine-quadratic term structure models: Toward the understanding of jumps in interest rate. In China International Conference in Finance, Chengdu, China.

Johannes, M. (2004). The statistical and economic role of jumps in continuoustime interest rate models. The Journal of Finance, 59(1), 227-260. http:// dx.doi.org/10.1111/j.1540-6321.2004.00632.x

Joslin, S. (2007). Pricing and hedging volatility risk in fixed income markets. Retrieved from https://www.researchgate.net/publication/228874222 _Pricing_and_hedging_volatility_risk_in_fixed_income_markets

Ludvigson, S. C., \& Ng, S. (2009). Macro factors in bond risk premia. Review of Financial Studies, 22(12), 5027-5067. http://dx.doi.org/10.1093/rfs/ hhp081

Pan, J. (2002). The jump-risk premia implicit in options: Evidence from an integrated time-series study. Journal of Financial Economics, 63(1), 3-50. http://dx.doi.org/10.1016/S0304-405X(01)00088-5

Wright, J. H., \& Zhou, H. (2009). Bond risk premia and realized jump risk. Journal of Banking \& Finance, 33(12), 2333-2345. http://dx.doi.org/ 10.1016/j.jbankfin.2009.06.010

\section{Appendix A Model and bond pricing}

Fix a probability space $\left(\Omega, \mathcal{F}_{t}, P\right)$ and a filtration $\left(\mathcal{F}_{t}\right)$, satisfying the usual conditions, and suppose that $X(t)$ is a Markov process in some state space $D \subset \mathbb{R}^{n}$, which evolves according to the following SDE:

$$
\mathrm{d} X(t)=\mu(X(t)) \mathrm{d} t+\sigma(X(t)) \mathrm{d} W(t)+\int_{\mathbb{R}^{n}} Y M(\mathrm{~d} y, \mathrm{~d} t)
$$


where $W$ is an $\left(\mathcal{F}_{t}\right)$-standard Brownian Motion in $\mathbb{R}^{n} ; \mu: D \rightarrow \mathbb{R}^{n}, \sigma: D \rightarrow \mathbb{R}^{n \times n}$, and $M$ is a Jump Measure, whose intensity is given by $\mu$. We suppose that this intensity measure can be decomposed in the following manner $\mu(\mathrm{d} y, \mathrm{~d} t)=$ $\nu(\mathrm{d} y) \mathrm{d} t=\lambda(X(t)) f(\mathrm{~d} y) \mathrm{d} t .^{5} f(y)$ is the jump fixed probability distribution on $\mathbb{R}^{n}$, and $\lambda(X(t))$ is the jump-intensity, for some $\lambda: D \rightarrow[0, \infty)$.

We fix an affine discount-rate function $R: D \rightarrow \mathbb{R}$ and impose an affine structure on $\mu, \sigma \sigma^{T}$, and $\lambda$ :

- $\mu(x)=K_{0}+K_{1} x$, for $K=\left(K_{0}, K_{1}\right) \in \mathbb{R}^{n} \times \mathbb{R}^{n \times n}$.

- $\left(\sigma(x) \sigma(x)^{T}\right)_{i j}=\left(H_{0}\right)_{i j}+\left(H_{1}\right)_{i j} x$, for $H=\left(H_{0}, H_{1}\right) \in \mathbb{R}^{n \times n} \times \mathbb{R}^{n \times n \times n}$.

- $\lambda(x)=l_{0}+l_{1} x$, for $l=\left(l_{0}, l_{1}\right) \in \mathbb{R} \times \mathbb{R}^{n}$.

- $R(x)=\rho_{0}+\rho_{1} x$, for $\rho=\left(\rho_{0}, \rho_{1}\right) \in \mathbb{R} \times \mathbb{R}^{n}$.

For $c \in \mathbb{C}^{n}$, define $\theta(c)=\int_{\mathbb{R}^{n}} e^{c y} f(\mathrm{~d} y)$. This jump transform $\theta$ determines the jump-size distribution. The coefficients $(K, H, l, \theta)$ of $X$ completely determine its distribution, given an initial condition $X(0)$.

\section{A.1 Princing Bonds}

The price of a default-free bonus with time to maturity $h$ is given by

$$
P(h, X(t))=\mathbb{E}^{\mathrm{Q}}\left(\exp \left\{-\int_{t}^{t+h} R(X(s)) \mathrm{d} s\right\} \mid \mathcal{F}_{t}\right) .
$$

Note that the expectation is taken under the risk-neutral measure $\mathbb{Q}$, where all discounted prices are martingals.

Under technical conditions the price of a bonus is given by

$$
P(h, x)=\exp \left\{A(h)+B(h)^{T} x\right\}
$$

where $A$ and $B$ satisfy the Ricatti's ODE:

$$
\begin{aligned}
& \dot{B}(h)=\rho_{1}-K_{1}^{T} B(h)-\frac{1}{2} B(h)^{T} H_{1} B(h)-l_{1}(\theta(B(h))-1), \\
& \dot{A}(h)=\rho_{0}-K_{0}^{T} B(h)-\frac{1}{2} B(h)^{T} H_{0} B(h)-l_{0}(\theta(B(h))-1),
\end{aligned}
$$

with boundary conditions $B(0)=0$ and $A(0)=0$. The $O D E(41)-(42)$ is easily obtained by an application of Ito's Lemma to the candidate solution

${ }^{5}$ With this hypothesis the Poisson Random Measure is equivalent to a Compound Poisson Process with intensity $\lambda(X(t))$ and jump-size fixed probability distribution $f(y)$. 
(40) of the bonus price (39) with the additional assumption that the expected instantaneous return of an asset is equal to the instantaneous risk free rate $R(x)$.

Bond yields can be found applying the following,

$$
y(h, t)=\bar{A}(h)+\bar{B}(h) \cdot X(t),
$$

where $\bar{A}(h)=-\frac{A(h)}{h}$ and $\bar{B}(h)=-\frac{B(h)}{h}$.

\section{Appendix B Specification of the market price of risk}

Equation (3) decomposes the Radon-Nikodym derivative in two terms: the change of measure of Poisson Processes, $\mathrm{dP} / \mathrm{d} \mathbb{P}^{\mathrm{MB}}$, and the change of measure of Brownian Motions, $\mathrm{d} \mathbb{P}^{\mathrm{MB}} / \mathrm{d} \mathbb{Q}$.

$$
\begin{array}{r}
\frac{\mathrm{d} \mathbb{P}}{\mathrm{d} \mathbb{P}^{\mathrm{MB}}}=\exp \left\{\int_{[0, T] \times \mathbb{R}^{n}}\left(\lambda^{\mathrm{Q}}(s) f^{\mathbb{Q}}(\mathrm{d} y, \mathrm{~d} s)-\lambda^{\mathbb{P}}(s) f^{\mathbb{P}}(\mathrm{d} y, \mathrm{~d} s)\right)\right. \\
\left.+\sum_{i=1}^{N_{T}} \phi\left(Y_{i}, \tau_{i}\right)\right\} .
\end{array}
$$

where $\phi\left(Y_{i}, \tau_{i}\right)=\ln \frac{\lambda^{\mathbb{P}}\left(\tau_{i}\right) \mathrm{d} f^{\mathbb{P}}\left(Y_{i}, \tau_{i}\right)}{\lambda^{\mathbb{Q}}\left(\tau_{i}\right) \mathrm{d} f^{\mathbb{Q}}\left(Y_{i}, \tau_{i}\right)}$ and $\tau_{i}$ is the $i$-th jump-time. Note that with this specification the intensity can be stochastic and jump-size distribution can be time varying, ${ }^{6}$

$$
\frac{\mathrm{d} \mathbb{P}^{\mathrm{MB}}}{\mathrm{d} \mathbb{Q}}=\mathcal{E}\left(-\int_{0}^{T} \gamma(s) \mathrm{d} s\right)
$$

where $\mathcal{E}$ is the stochastic exponential defined as $\mathcal{E}(X(t))=\exp \{X(t)-[X, X](t)\}$, $[X, X]$ is the total quadratic-variation process, and $\gamma(t)$ is the market price of risk of the form Extended Affine (see Cheridito, Filipović, \& Kimmel, 2007):

$$
\gamma(t)=\left(\sigma(X(t)) \sigma(X(t))^{T}\right)^{-1}\left(\left(K_{0}^{\mathbb{P}}-K_{0}^{\mathbb{Q}}\right)+\left(K_{1}^{\mathbb{P}}-K_{1}^{\mathbb{Q}}\right) X(t)\right) .
$$

${ }^{6}$ If the Poisson Random Measure is a Compound Poisson Process with constant intensity and invariant jump-size distribution, then the Radon-Nikodym derivative is

$$
\frac{\mathrm{d} \mathbb{P}}{\mathrm{d} \mathbb{P}^{\mathrm{MB}}}=\exp \left\{T\left(\lambda^{\mathbb{Q}}-\lambda^{\mathbb{P}}\right)+\sum^{N_{T}} \ln \frac{\mathrm{d} \nu^{\mathbb{P}}(Y)}{\mathrm{d} \nu^{\mathbb{Q}}(Y)}\right\} .
$$




\section{Appendix C Specification of the models}

\section{C.1 A30}

$$
\begin{array}{rcc}
K_{1}^{P}=\left[\begin{array}{ccc}
K_{1,11}^{P} & 0 & 0 \\
K_{1,21}^{P} & K_{1,22}^{P} & 0 \\
K_{1,31}^{P} & K_{1,32}^{P} & K_{1,33}^{P}
\end{array}\right], & K_{1}^{Q}=\left[\begin{array}{ccc}
K_{1,11}^{Q} & 0 & 0 \\
K_{1,21}^{Q} & K_{1,22}^{Q} & 0 \\
K_{1,31}^{Q} & K_{1,32}^{Q} & K_{1,33}^{Q}
\end{array}\right] \\
K_{0}^{P}=\left[\begin{array}{l}
0 \\
0 \\
0
\end{array}\right], & K_{0}^{Q}=\left[\begin{array}{c}
K_{0,1}^{Q} \\
K_{0,2}^{Q} \\
K_{0,3}^{Q}
\end{array}\right]
\end{array}
$$

$\rho_{0} \in \Re ; \rho_{1}=[1,1,1] ; H_{0, i j} \in \Re_{+}$for $i=j$ and 0 otherwise; $H_{1, i j}=[0,0,0]$ for $i, j=1,2,3 ; l_{0}^{Q}=l_{0}^{P}=0$, and $l_{1}^{Q}=l_{1}^{P}=[0,0,0]$.

\section{C.2 A30J}

Equal to A30, except for $l_{0}^{i} \in \Re_{+}$for $i=Q, P$, and $f^{i}(y)$ is gaussian with mean $\mu_{J}^{i}$ and variance $\Sigma_{J}^{i}$ for $i=Q, P$.

$$
\begin{array}{ccc}
\Sigma_{J}^{P}=\left[\begin{array}{ccc}
0 & 0 & 0 \\
0 & 0 & 0 \\
0 & 0 & \Sigma_{J, 33}^{P}
\end{array}\right], & \Sigma_{J}^{Q}=\left[\begin{array}{ccc}
0 & 0 & 0 \\
0 & 0 & 0 \\
0 & 0 & \Sigma_{J, 33}^{Q}
\end{array}\right] \\
\mu_{J}^{P}=\left[\begin{array}{c}
0 \\
0 \\
\mu_{J, 3}^{P}
\end{array}\right], & \mu_{J}^{Q}=\left[\begin{array}{c}
0 \\
0 \\
\mu_{J, 3}^{Q}
\end{array}\right]
\end{array}
$$

$\mu_{J, 3}^{i} \in \Re$ and variance $\Sigma_{J, 33}^{i} \in \Re_{+}$for $i=Q, P$.

\section{C.3 A30JT}

Exactly the same as A30J, but with $l_{1}^{i} \in \Re_{+}^{3}$ for $i=Q, P$. 